\title{
SCIDic
}

International Journal of Dentistry and Oral Science (IJDOS)

ISSN: 2377-8075

\section{Neurofibromatosis type I with an Rare Oral Manifestation}

Case Report

Cedraz de Oliveira $\mathrm{M}^{1}$, Oliveira dos Santos Freitas $\mathrm{C}^{1}$, Santos Pereira Ramos $\mathrm{ME}^{2}$, de Carvalho Freitas Ramos $\mathrm{T}^{3}$, Freitas VS ${ }^{3}$, Martins Cerqueira JD ${ }^{4 *}$

'Student of the Course of Dentistry of the Feira de Santana State University, Feira de Santana, Bahia, Brazil.

${ }^{2}$ Professor of Surgery at the Odontology course at the Feira de Santana State University, Feira de Santana, Bahia, Brazil.

${ }^{3}$ Professor of Pathology at the Odontology course at the Feira de Santana State University, Feira de Santana, Bahia, Brazil.

${ }^{4}$ Professor of Collective Health Course in Dentistry at the Feira de Santana State University, Feira de Santana, Bahia, Brazil.

\section{Abstract}

Neurofibromatosis type I is one of the most common genetic diseases and it may have oral manifestation.

Objetive: To report a case about a 33 years old female patient diagnosed with neurofibromatosis type I in the skin.

Case Report: In the extraoral clinical examination were observed subcutaneous nodules and café-au-lait spots, in different sizes around the body. In the intraoral examination was diagnosed the presence of rounded nodule in the hard palate. The excisional biopsy of the lesion was performed and the histopathological examination suggested a diagnosis of neurofibroma. Conclusion: The case report emphasizes the importance of a detailed anamnesis and a thorough physical examination, so that systemic diseases can be considered in oral diagnosis.

Keywords: Neurofibromatosis; Neurofibroma; Mouth.

\section{Introduction}

Neurofibromatosis type I (NF1), classically described by Friedrich Daniel von Recklinghausen in 1882, is the most common autosomal genetic disease with dominant inheritance of the human species. The prevalence of NF1 is one case per 3,000 births, and may be appear at birth or late, there is no evidence of predilection for gender or ethnicity [1].

This neuro-skin disorder is caused by a mutation of the NF1 gene located on the long arm of the chromosome 17q112, causing the loss of function of the protein neurobromina, thus leading to multiple manifestations of the phenotype of NF1 [2].

Clinically, there are seven major components of the syndrome, including the presence of cafe-au-lait spots, two or more neurofibromas of any type or a plexiform neurofibroma, freckles in the axillary or inguinal region, optic gliomas, two or more Lish nodules, bone lesions, these are the primary degree relative with NF1 according to the preceded criteria. If the individual has at least two of these characteristics, he can be diagnosed with NF1 [1-3].

Despite being more prevalent in skin, NF1 may be manifested in the oral cavity in about $10 \%$ of the cases, particularly in the tongue $[3,4]$. However, changes such as macroglossia, increased of fungiform papilla and mandibular foramen, unilateral or bilateral intraosseous lesion and delayed tooth eruption can also be found in patients with NF1 [5].

The prognosis of NF1 is unpredictable, showing the possibility of various complications [2]. The malignant transformation of NF1 has been reported, mainly evolving to malignant neoplasm of peripheral nerve sheath and neurofibrosarcoma [6, 7]. However, the malignant transformation is poorly understood, and in particular the mutation in the p53 gene on chromosome $17 \mathrm{p}$ is associated with this progression [7].

Thus, the aim of this article is to present one case of a classic neurofibromatosis type 1 , with clinical evidence similar to the literature, but showing an unusual oral manifestation.

*Corresponding Author:

Joana Dourado Martins Cerqueira,

Professor of collective health course in Dentistry at the Feira de Santana State University, Feira de Santana, Bahia, Brazil..

Tel: (55) (75)3161-8248

Fax: 07536618089

E-mail: martinsjoana_1@hotmail.com

Received: July 07, 2017

Accepted: August 21, 2017

Published: August 24, 2017

Citation: Cedraz de Oliveira M, Oliveira dos Santos Freitas C, Santos Pereira Ramos ME, de Carvalho Freitas Ramos T, Freitas VS, Martins Cerqueira JD. Neurofibromatosis type I with an Rare Oral Manifestation. Int J Dentistry Oral Sci. 2017;4(8):513-516. doi: http://dx.doi.org/10.19070/2377-8075-17000101

Copyright: Martins Cerqueira JD ${ }^{\circ}$ 2017. This is an open-access article distributed under the terms of the Creative Commons Attribution License, which permits unrestricted use, distribution and reproduction in any medium, provided the original author and source are credited. 


\section{Case Report}

A 33 years old female patient, melanoderma, sought the Dentistry Clinic of Integrated Studies XIV at the State University of Feira de Santana - Bahia, complaining about "the presence of lumps in the roof of the mouth."

In the an amnesis the patient reported the presence of nodules around the body since birth, and a surgery to remove a nodule on the right side of the face about 15 years ago, which removed the ear (Figure 1). The histopathological diagnosis of the lesion localized in her face was neurofibroma. Also, the patient reported the appearance of nodule in the mouth for a year and two months ago (Figure 2). In the medical history, the patient denied having health problems and said that there is no other case of neurofibromatosis type 1 in her family.

In the extraoral clinical examination was observed facial asymmetry (Figure 3), scoliosis, short stature, subcutaneous nodules of different sizes around the body, the plexiform neurofibroma in the arms (Figure 4) and café-au-lait spots on the limbs lower (Figure 5). The intraoral examination showed macroglossia, an increase of fungiform papillae and the presence of rounded nodules in the hard palate measuring about $5 \mathrm{~mm}$, showing regular contour, pink color, soft consistency and exophytic growth.

An excisional biopsy of the lesion and the histopathologic examination was performed and it showed mesenchymal tissue fragments with fusiform cells and corrugated core arranged in interlocking beams, permeated by connective tissue stroma composed of delicate collagen bundles (Figure 6), suggesting the diagnosis of neurofibroma.

This case has the approval of the ethics committee with CAAE: 0086.059.000-08 the information in this article has permission from the patient to divulgation.

\section{Discussion}

NF1 is an autosomal genetic disease with dominant inheritance; it is common to have more than one case in the same family [1]. In this case report, the patient is unaware of another family member carrier of the disease.

The initial suspected diagnosis was based on clinical manifesta-

Figure 1. Scar of the surgery of a neurofibroma which in the face, when she had the excision of the ear.

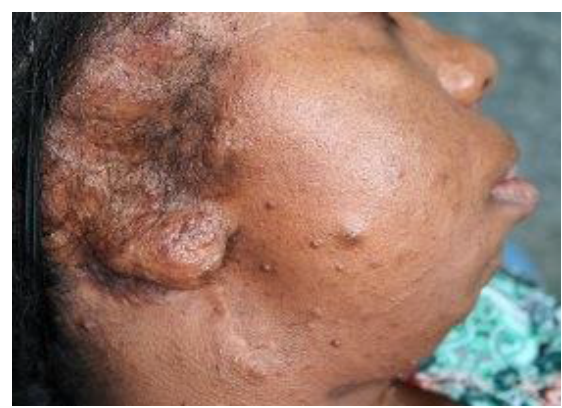

Figure 2. Lesion on the palate and tongue appeared about a year and two months.

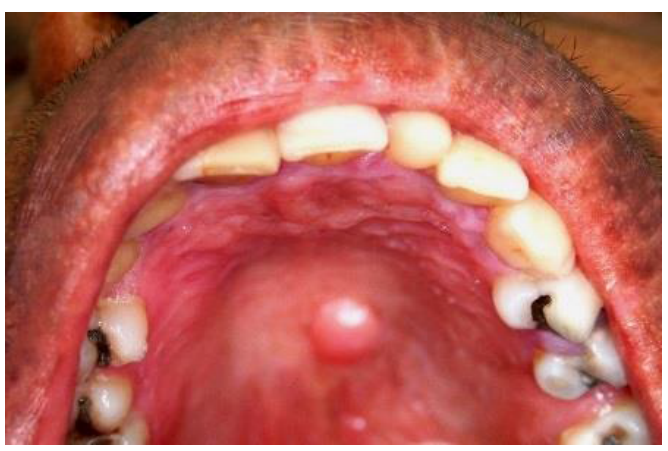

Figure 3. Facial asymmetry observed in the extraoral examination, it is showing the presence of subcutêneos nodes on the neck.

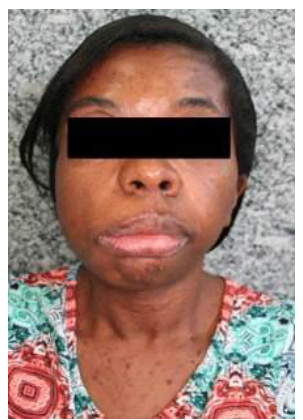


Figure 4. Plexiform neurofibromas located in the upper limbs.

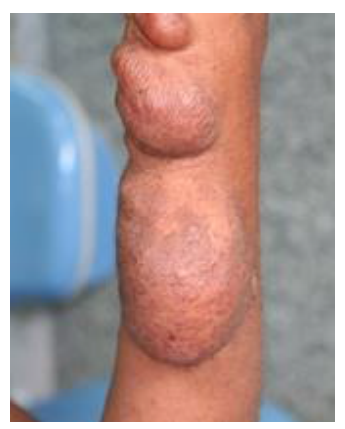

Figure 5. Café-au-lait spots in the lower limbs.

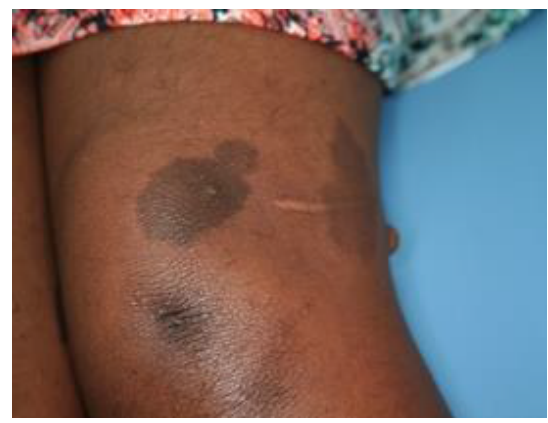

Figure 6. Macro photography of the neoplastic proliferation of fusiform fibroblast wavy nuclei in submucosal region $(\mathrm{H} / \mathrm{E}, 4 \mathrm{X})$.

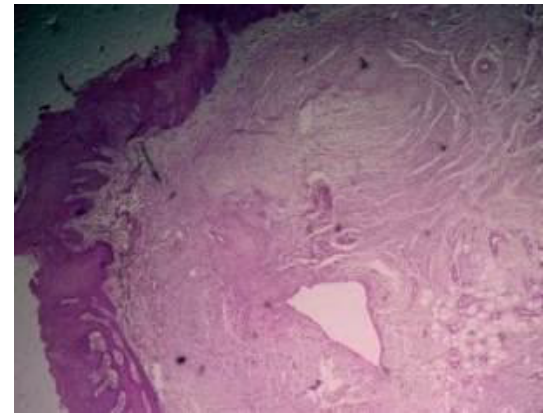

tion of the disease and was confirmed by histopathology examination, in accordance with the protocol recommended by the literature [9].

The clinical findings in the patient corroborate with those mentioned by the literature, once she has the presence of cafe-au-lait spots in the lower limbs, as well as plexiform neurofibroma in the upper limbs, which is a pathognomonic characteristics for this disease [1-3]. It was also noticed a hemi facial hypertrophy, scoliosis and short stature [9].

The first diagnosis of NF1 occurred when the patient was between the first and second decade of life after a surgical removal of a lesion in the face. The diagnosis of this disease in common at this age group, because during puberty occurs an exacerbation in the number and size of neurofibromas, which leads the patient to seek a professional follow-up [3].

The oral manifestation of NF1 is controversial. Some authors admit this is a common expression of the disease, which occurs in $72 \%$ of cases [6], others authors believe this is rare, affecting only $10 \%$ to $26 \%$ of cases [1]. In this case report the oral manifestations occurred three decades after the appearance of the characteristics in the skin, showing neurofibromas on the palate, which is an unusual site [4]. In the intraoral examination was also observed macroglossia, which is in accordance with the aspects commonly found for the disease in the mouth [9].

Currently, the patient is being followed in a close proservation, since some authors admit the possibility of malignant transformation of NF1. This transformation remains controversial in the literature, ranging from 3 to $5 \%$ (9) or affecting up to $26 \%$ of cases [10]. This disparity suggests a certain degree of unaware about the malignant mechanism of NF1, being essential that more studies be conducted about this topic.

The treatment of choice is limited to provide a better quality of life for the individual affected by NF1. The surgical excision of the lesions is indicated in the most cases [9], which was the proposed treatment for this case. Since the aesthetic deformity that the neurofibromas can provide, it may result in a psychosocial impairment of the patient, so that it is necessary a multidisciplinary follow-up [3]. In this case, the patient showed up quite shy, reporting she never did any kind of work throughout life, so it was proposed to her a psychotherapeutic therapy as a treatment. 


\section{Conclusion}

As it has been seen, the characteristics findings in this case are similar to those reported in the literature, differing only by the rare anatomical location on the palate. Also, it is important to highlight the importance of a detailed anamnesis and physical examination, once systemic diseases may have oral manifestations. It is important to emphasize the necessity of a follow up in these patients diagnosed with oral NF1, because the malignant transformation is possible and the rates of recurrence of the lesions in skin and mouth are high.

\section{References}

[1]. Dova S, Ktenidis K, Karkos P, Blioskas S, Psillas G, Iliadis A, et al. rare case of a spontaneous neck hematoma in a patient with type 1 neurofibromatosis. Auris Nasus Larynx. 2016 Oct;43(5):591-594 PubMed PMID: 27061148.

[2]. Iannuzzi S, Albaret JM, Chignac C, Faure-Marie N, Barry I, Karsenty C, et al. Motor impairment in children with Neurofibromatosis type 1: Effect of the comorbidity with language disorders. Brain Dev. 2016 Feb;38(2):181-7. PubMed PMID: 26321374

[3]. Ghalayani P, Saberi Z, Sardari F. Neurofibromatosis type I (von Reckling- hausen's disease): A family case report and literature review. Dent Res J (Isfahan). 2012 Jul 9(4):483-8.PubMed PMID: 23162593.

[4]. Vuity D, Németh Z, Bogdán S. A 40-year-old palatal neurofibroma. A case reports. Fogorv Sz. 2013 Mar 106(1):3-6.PubMed PMID:23650755.

[5]. Hirbe AC, Pekmezci M, Dahiya S, Apicelli AJ, Van Tine BA, Perry A, et al. Braf V600E mutation in sporadic and neurofibromatosis type 1-related malignant peripheral nerve sheath tumors Neuro Oncol. 2014 Mar 16(3):466467. PubMed PMID: 24366910.

[6]. Chander V, Rao RS, Sekhar G, Raja A, Sridevi M. Recurrent Diffuse Neurofibroma of Nose Associated with Neurofibromatosis Type 1: A Rare Case Report with Review of Literature. Indian J Dermatol. 2015 Nov-Dec 60(6):573-560.PubMed PMID: 26677270.

[7]. Patil, K.; Mahima, VG; Shetty, SK; Lahari, K. Facial plexiform neurofibroma in a child with neurofibromatosis type I: a case report. J Indian Soc Pedod Prev Dent. 2007 Mar 25(1):30-35. PubMed PMID: 17456965.

[8]. Shapiro SD, Abramovitch K, Van Dis ML, Skoczylas LJ, Langlais RP, Jorgenson RJ, et al. Neurofibromatosis: oral and radiographic manifestations. Oral Surg Oral Med Oral Pathol. 1984 Oct 58(4):493-498. PubMed PMID: 6436765

[9]. Holtzman, L. Radiographic manifestation and treatment considerations in a case of multiple neurofibromatosis. J Endod. 1998 Jun 24(6):442-443. PubMed PMID: 9693592.

[10]. Bongiorno, MR; Pistone, G; Aricò, M. Manifestations of the tongue in Neurofibromatosis type 1. Oral Dis. 2006 Mar 12(2):125-134. PubMed PMID:16476032. 